\title{
1 Prediction of intensive care unit mortality based on missing events
}

2 Tatsuma Shoji (1) ${ }^{* 1, * 2}$, Hiroshi Yonekura (2) ${ }^{* 2}$, Sato Yoshiharu (1), Yohei Kawasaki (3)

$3 * 1$ : Corresponding Author; *2: Contributed equally

4

5 (1) DNA Chip Research Inc.

6 1-15-1 Kaigan, Suzue Baydium 5F, Minato-ku, Tokyo 105-0022, Japan

8 (2) Department of Anesthesiology and Pain Medicine, Fujita Health University Bantane

9 Hospital

10 2-6-10 Otoubashi, Nakagawa-ku, Nagoya City, Aichi, 454-8509, Japan

12 (3) Faculty of Nursing, Japan Red Cross College of Nursing, Tokyo, Japan

13 4-1-3 Hiroo, Shibuya-ku, Tokyo 150-0012, Japan

15 E-mail: Shoji Tatsuma: t-shoji@dna-chip.co.jp

16 Yonekura Hiroshi: hiroshi.yonekura@fujita-hu.ac.jp

17 Sato Yoshiharu: yo-sato@dna-chip.co.jp

18 Kawashiki yohei: ykawasaki@ chiba-u.jp 


\section{Abstract \\ 20 Background}

21 The increasing availability of electronic health records has made it possible to

22 construct and implement models for predicting intensive care unit (ICU) mortality using

23 machine learning. However, the algorithms used are not clearly described, and the

24 performance of the model remains low owing to several missing values, which is

25 unavoidable in big databases.

\section{Methods}

27 We developed an algorithm for subgrouping patients based on missing event

28 patterns using the Philips eICU Research Institute (eRI) database as an example. The

29 eRI database contains data associated with 200,859 ICU admissions from many

30 hospitals (>400) and is freely available. We then constructed a model for each subgroup

31 using random forest classifiers and integrated the models. Finally, we compared the

32 performance of the integrated model with the Acute Physiology and Chronic Health

33 Evaluation (APACHE) scoring system, one of the best known predictors of patient

34 mortality, and the imputation approach-based model.

\section{Results}


36 Subgrouping and patient mortality prediction were separately performed on

37 two groups: the sepsis group (the ICU admission diagnosis of which is sepsis) and the

38 non-sepsis group (a complementary subset of the sepsis group). The subgrouping

39 algorithm identified a unique, clinically interpretable missing event patterns and divided

40 the sepsis and non-sepsis groups into five and seven subgroups, respectively. The

41 integrated model, which comprises five models for the sepsis group or seven models for

42 the non-sepsis group, greatly outperformed the APACHE IV or IVa, with an area under

43 the receiver operating characteristic (AUROC) of 0.91 (95\% confidence interval

44 0.89-0.92) compared with $0.79(0.76-0.81)$ for the APACHE system in the sepsis group

45 and an AUROC of $0.90(0.89-0.91)$ compared with $0.86(0.85-0.87)$ in the non-sepsis

46 group. Moreover, our model outperformed the imputation approach-based model, which

47 had an AUROC of $0.85(0.83-0.87)$ and $0.87(0.86-0.88)$ in the sepsis and non-sepsis

48 groups, respectively.

\section{Conclusions}

50 We developed a method to predict patient mortality based on missing event

51 patterns. Our method more accurately predicts patient mortality than others. Our results

52 indicate that subgrouping, based on missing event patterns, instead of imputation is

53 essential and effective for machine learning against patient heterogeneity. 


\section{Trial registration}

$55 \quad$ Not applicable.

\section{Keywords}

57 ICU, patient mortality, Acute Physiology and Chronic Health Evaluation scoring,

58 machine learning, missing values

\section{Background}

61 Accurate prognostication is central to medicine [1] and at the heart of clinical

62 decision-making. Sepsis is a systemic response to infection, with the highest mortality

63 rate in the field of intensive care. For nearly a decade, sepsis-related mortality has

64 remained at 20\%-30\%, with unsatisfactory improvements [2]. Prognosis remains a

65 challenge for physicians because of the high heterogeneity of clinical phenotypes [3].

66 Because accurate diagnoses can improve the physician's decision-making abilities, it is

67 one of the essence for medical practice of sepsis to improve prognosis accuracy [4].

68 Most large prognostic studies have developed clinical scoring systems for

69 objective risk stratification in the early phase of hospital admission using physiological

70 measurements, medical history, and demographics to predict the likelihood of survival

71 [5]. Among these scoring systems, Acute Physiology and Chronic Health Evaluation 
72 (APACHE) scoring [6] is one of the best known, which has been validated for

73 application at approximately $24 \mathrm{~h}$ after intensive care unit (ICU) admission. The

74 APACHE scoring system generates a point score based on worst values of 12 variables

75 during the initial $24 \mathrm{~h}$ after ICU admission. The APACHE II score was published in

761985 [5]; APACHE IV and IVa are the latest versions [7]. Built on the study of a more

77 recent patient population and standard-of-care, APACHE IV or IVa are recommended

78 as scoring systems over APACHE II and III. However, these indicators lack the

79 precision required for use at the individual level. Therefore, efforts have been made to

80 increase the performance of these indicators through the use of computational

81 techniques, such as machine learning.

82 Machine learning classifiers may be advantageous for outcome prediction

83 because they can handle large numbers of variables and learn non-linearities [8,9].

84 Random forest is an example of modern machine learning algorithms [10]. Machine

85 learning requires large volumes of data and generating a complex model. However, the

86 increasing availability of electronic health records has made constructing and

87 implementing the models possible. The availability of large training sets $[11,12]$ has

88 made investigation of such approaches feasible. Studies on the application of machine

89 learning to intensive care datasets have been performed $[13,14,15,16,17,18,19,20$, 
90 21]. Traditional scoring systems were outperformed by machine learning approaches in

91 mortality prediction in medical ICUs. However, the algorithms are not clearly described

92 and the produced models or databases are available commercially.

93 The Philips eICU Research Institute (eRI) is a non-profit institute established

94 by Phillips that is governed by customers [22]. This freely accessible critical care

95 dataset spans more than a decade and contains detailed information about individual

96 patient care, including time-stamped, nurse-verified physiological measurements. As a

97 distinctive feature, datasets are donated by $>400$, including teaching and non-teaching,

98 hospitals. However, the eRI, which comprises 31 files according to clinical categories,

99 includes much missing data. This makes the application of machine learning with eRI

100 challenging.

101 In this study, we revealed unique characteristics of the distribution of missing

102 values in the eRI database. By taking advantage of this characteristic, we successfully

103 developed a more accurate and interpretable model against the APACHE system.

104 Accurate prediction of patient state is critical in the critical care field. To address this

105 problem, we propose the "missing-event-based prediction," a new method for predicting

106 ICU mortality. 


\section{Methods}

109 Dataset used in this study

$110 \quad$ We used the eRI

111 (https://www.usa.philips.com/healthcare/solutions/enterprise-telehealth/eri)

112 database, which is an open database and provides anonymous data, including

113 demographic information, vital signs measurements, laboratory test results, drug

114 information, procedural information, fluid balance reports, hospital length of stay data,

115 and data on in-hospital mortality, donated by $>400$ member institutions. The eRI

116 contains data associated with 200859 ICU admissions with more than 100 variables. In

117 this study, we carefully selected 58 clinically important variables to construct a model,

118 which is easy to interpret clinically. Selected variables are summarized in Table S1 (see

119 Additional file 1). Briefly, the variables were as follows: (I) Listed from laboratory

120 measurements, including white blood cell count, hematocrit, bilirubin, creatinine,

121 sodium, albumin, blood urea nitrogen, glucose, arterial $\mathrm{pH}$, fraction of inspired oxygen,

122 arterial oxygen pressure, and arterial blood carbon dioxide pressure. (II) Listed from

123 routine charted data, including temperature, respiratory rate, heart rate, mean arterial

124 blood pressure, urine output, and Glasgow Coma Scale (including score for eye, motor,

125 and verbal responses). (III) Listed from information taken at the time of ICU admission, 
medRxiv preprint doi: https://doi.org/10.1101/2021.02.28.21252249; this version posted March 2, 2021. The copyright holder for this preprint

(which was not certified by peer review) is the author/funder, who has granted medRxiv a license to display the preprint in perpetuity.

All rights reserved. No reuse allowed without permission.

126 including age, gender, height, weight, time from hospitalization to ICU admission, type

127 of hospital, bed count of the hospital, hospital ID, and diagnoses names at ICU

128 admission. (IV) Comorbidities, including myocardial infarction within 6

129 months, diabetes, hepatic failure, dialysis, immunosuppressive disease, lymphoma,

130 leukemia, metastatic cancer, cirrhosis, acquired immune deficiency syndrome,

131 and history of intubation and mechanical ventilation. Intervention required at admission,

132 including catheter intervention for myocardial infarction, coronary artery bypass

133 grafting with or without internal thoracic artery grafts, and use of thrombolytics. (V)

134 APACHE scoring system, including not only the APACHE score but also actual ICU

135 and in-hospital mortality, predicted ICU and in-hospital mortality, length of ICU stay,

136 length of hospital stay, and ventilation duration. Among these variables, diagnoses

137 names at ICU admission were used to define the sepsis or non-sepsis group (see the

138 Definition of sepsis and non-sepsis groups in Methods section for details), actual ICU

139 and in-hospital mortality were used as response variables to construct the models,

140 predicted ICU and in-hospital mortality using the APACHE IV or IVa were used as

141 benchmarks against our model, and others (53 parameters in total) were used as

142 explanatory variables for machine learning. 


\section{Definition of sepsis and non-sepsis groups}

145 Inclusion criteria for the sepsis group were as follows: (I) extraction by

146 diagnoses names at ICU admission, namely "Sepsis, cutaneous/soft tissue," "Sepsis, GI,"

147 “Sepsis, gynecologic," "Sepsis, other," "Sepsis, pulmonary," "Sepsis, renal/UTI

148 (including bladder)," and "Sepsis, unknown;" (II) selection by documentation of

149 prognosis; and (III) exclusion by cases with any missing data in Acute Physiology Score

150 (APS)-related variables and prognosis information. Inclusion criteria for the non-sepsis

151 group were almost the same as the one used for the sepsis group. Briefly, the

152 complementary subset of (I) was first selected. Then, (II) and (III) were applied to the

153 resulting subset. Thus, 4226 and 23170 cases were defined as sepsis and non-sepsis

154 groups, respectively. To reproduce our results and access patient lists, follow the jupyter

155 notebook at

156 https://github.com/tatsumashoji/ICU/1_the_sepsis_group_and_non_sepsis_group.ipynb.

158 Subgrouping based on missing data

159 Subgroups were defined according to the diagram shown in Fig. S1 (see

160 Additional file 2). Briefly, (I) patient lists, containing no missing data for any pattern of

16152 variables (derived from 53 variables) were first generated. Then, (II) the patient list, 
162 which had a size not too small and not too large among 53 lists, was defined as

163 subgroup \#1. For the other subgroups, we repeated (I) and (II) with the other patients.

164 To reproduce this subgrouping, follow the jupyter notebook opened at

165 https://github.com/tatsumashoji/ICU/2_subgrouping_sepsis.ipynb for the sepsis group

166 and https://github.com/tatsumashoji/ICU/3_subgrouping_non_sepsis.ipynb for the

167 non-sepsis group.

169 Generation and performance of our model

170 To construct the model for each group, we used the random forest classifier

171 implemented with "scikit-learn (0.24.1)" [23]. Briefly, we first selected 80\% data as a

172 training dataset for each group so that the ratio of "ALIVE" and "EXPIRED" cases

173 were the same between the two datasets. After hyperparameters for the random forest

174 were determined using the grid search algorithm, the actual model was generated, and

175 the mean and standard deviation of accuracy were checked through 5-fold

176 cross-validation (see Table S2-S5 in Additional file 1). Finally, patient mortalities in the

177 test dataset were predicted by the generated model and compared to those from

178 APACHE IV or IVa by drawing receiver operating characteristic (ROC) curves and

179 calculating the area under the ROC (AUROC). The confidence intervals for the 
180 AUROC were calculated as described [24]. For calibration plots, we used the module

181 “sklearn.calibration.calibration_curve." All results in Fig. 2 can be reproduced by

182 running “https://github.com/tatsumashoji/ICU/4_sepsis_prediction.ipynb.”

183

184 Imputation of missing values

185 For imputation of missing values, we used multivariate imputation algorithms

186 implemented with "sklearn.impute.IterativeImputer," which uses the entire set of

187 available feature dimensions to estimate missing values. We used the 0.24 .1 version of

188 scikit-learn. To reproduce results shown in Fig. 3, follow the jupyter notebook opened

189 at https://github.com/tatsumashoji/ICU/5_imputation.ipynb.

190

191 Results

192 Heterogeneity of the sepsis group

193 Machine learning fails to predict the outcomes if input data consist of more

194 than two populations. Subgrouping of input data is essential before constructing the

195 model using machine learning. Therefore, we first investigated the distribution of each

196 parameter in the sepsis group. The histogram in Fig. 1a shows the distribution of some

197 variables recorded in "apacheAps Var.csv", which contains the variables used to 
198 calculate the Acute Physiology Score (APS) III for patients. More than two peaks were

199 observed, indicating the necessity for subgrouping the sepsis group. Importantly, cases

200 with missing data on any variable are excluded from the histograms in Fig. 1a. Thus, to

201 examine how missing values were distributed in the sepsis group, correlation

202 coefficients were calculated for all possible combinations of two variables after missing

203 data were replaced with 0 and others with 1 (Fig. 1b). Surprisingly, perfect correlations

204 were observed in some pairs in addition to the diagonal line. This suggests that missing

205 events occur depending on other missing events. Clinically, this can be explained by

206 different histories and backgrounds in the sepsis group. Thus, missing events contain

207 information and may play an important role in subgrouping the sepsis group.

209 Machine learning combining missing-event-based subgrouping approach

210 outperforms APACHE

211 The unique distribution of missing values in the sepsis group led us to divide

212 the group before machine learning. To account for the pattern of missing events when

213 dividing the sepsis group, we defined subgroups such that each subgroup had the same

214 missing pattern while the number of subgroups could as small as possible and the size

215 of each subgroup could be as large as possible (Fig. 2a). For details, see the Methods 
medRxiv preprint doi: https://doi.org/10.1101/2021.02.28.21252249; this version posted March 2, 2021. The copyright holder for this preprint

(which was not certified by peer review) is the author/funder, who has granted medRxiv a license to display the preprint in perpetuity.

All rights reserved. No reuse allowed without permission.

216 section. After defining five subgroups, we constructed models for each subgroup based

217 on the random forest algorithm and calculated patient mortality. Then, we assessed the

218 performance of each model by calculating AUROC and compared them to those from

219 the APACHE IV or IVa system (Fig. 2b). Our model outperformed the APACHE

220 systems, especially when integrating subgroups. Moreover, our model was more

221 successful that APACHE in distinguishing patient mortality (Fig. 2c). Furthermore,

222 results from calibration plots supported the ability of our model to predict patient

223 mortality (Fig. 2d). Our model tends to output patient mortality higher than actual,

224 whereas the APACHE system does not, indicating that the APACHE system

225 underestimates patients' mortality.

227 Comparison with the imputation approach

228 A typical way of handling missing data is to impute them. Therefore, we

229 constructed the model completely same as the way taken in Fig. 2 after imputing the

230 dataset first, then compared to our model. Although the performance when imputed was

231 slightly higher than the APACHE systems, it still remained lower than our method (Fig.

232 3a), indicating that missing events are important information and our method, which

233 accounts for the pattern of missing events, is reasonable. The imputation approach 
234 outperforms the approach of the APACHE system but does perform as well as our

235 model, as confirmed using the scatter plot (Fig. 3b). Our model distinguished patient

236 mortality most precisely among all four models. Moreover, analyses of calibration plots

237 supported the observation that our model is the most conservative, and thus, safer,

238 because the model based on the imputation approach estimated lower patient mortality

239 compared with our model (Fig. 3c).

\section{Application to the non-sepsis group}

243 non-sepsis group defined in the Methods section. We first generated seven subgroups

244 using the same algorithm used for the sepsis group (Fig. 4a) and constructed models for

245 each subgroup. Then, we compared the performance of the four models, namely our

246 model, APACHE IV, APACHE IVa, and the model based on the imputation approach

247 (Fig. 4b). Surprisingly, the distribution of missing values in the non-sepsis group was

248 almost the same as that in the sepsis group, indicating that our subgrouping algorithm

249 could be used for any group in addition to the sepsis group, and the performance of our

250 model was the highest among all four models. Scatter plots and calibration plots also

251 supported our model (Fig. S4, S5, see Additional file 2). These results strongly suggest 
medRxiv preprint doi: https://doi.org/10.1101/2021.02.28.21252249; this version posted March 2, 2021. The copyright holder for this preprint

(which was not certified by peer review) is the author/funder, who has granted medRxiv a license to display the preprint in perpetuity.

All rights reserved. No reuse allowed without permission.

252 that missing events themselves are essential when predicting patient mortality in the

253 ICU.

254

255 Discussion

256 The main objective of this work was to present a more accurate and clinically

257 interpretable model for predicting patient mortality in the ICU and show the

258 effectiveness and potential of the missing-event-based prediction method. Our analysis

259 of the eRI confirmed that the larger the database across hospitals, the more the

260 heterogeneity (Fig. 1a). This is known as the domain shift problem, where traditional

261 models, including the APACHE system, developed in one geographical region or

262 healthcare system lose their ability to discriminate when applied outside of learning data.

263 The effect of prediction accuracy by volume and diversity of missing events clearly

264 increases in big data. We demonstrated the advantage of using a missing-event-based

265 method through comprehensive analysis and presented a more accurate and

266 interpretable model than others.

267 In the eRI database, missing events occurred depending on other missing

268 events (Fig. 1b). Thus, missing events were not random. Such cases would not be rare in

269 big data because the larger the dataset, the more complex the missing events. If missing 
medRxiv preprint doi: https://doi.org/10.1101/2021.02.28.21252249; this version posted March 2, 2021. The copyright holder for this preprint

(which was not certified by peer review) is the author/funder, who has granted medRxiv a license to display the preprint in perpetuity.

All rights reserved. No reuse allowed without permission.

270 event was not regarded "missing at random," the simple imputation of missing values

271 would not be appropriate [25]. For example, Meiring et al. developed an algorithm to

272 predict mortality over time in the ICU using CCHIC (a UK database) [20]. They

273 imputed missing values using predictive mean matching through parallel

274 implementation of multiple imputation by chained equations [26]. In this report, the

275 discriminative power of the APACHE II score to predict outcomes on subsequent days

276 reduced considerably. Moreover, Meiring et al. indicated that the longer the ICU stay,

277 the smaller the size of the dataset, and therefore, an increase in the proportion of

278 missing values. Thus, the weight of missing values impaired the accuracy of prediction.

279 Handling missing values is important to generate a powerful model workable on

280 complex clinical courses. Thus, our missing-event-based method is reasonable. Because

281 the subgrouping pattern in this study was humanly impossible to detect, although easy

282 to understand, our subgrouping algorithm could be the new de facto standard of

283 database prescreening when constructing an accurate and interpretable model through

284 machine learning using big data, including missing values. Our subgrouping algorithm

285 can recognize missing events as an "informative missingness" instead of a dirty record,

286 which big data cannot avoid including. 
medRxiv preprint doi: https://doi.org/10.1101/2021.02.28.21252249; this version posted March 2, 2021. The copyright holder for this preprint

(which was not certified by peer review) is the author/funder, who has granted medRxiv a license to display the preprint in perpetuity.

All rights reserved. No reuse allowed without permission.

287 For constructing the model based on the random forest algorithm, we selected

28853 variables as explanatory variables so that they were closely related to the APACHE

289 system. This selection is an important aspect of the interpretability of our model

290 because the APACHE system consists of clinically used variables. This feature is quite

291 important because the interpretable model addresses the problem of the "black box",

292 which has hindered the use of this model as a clinical tool [27, 28, 29]. Given that our

293 model greatly outperformed the APACHE system, it can be considered a developed

294 version of the APACHE system, which detects the presence of more complex

295 interactions between covariates, leading to optimization both of clinical usability and

296 discrimination ability.

297 As tested with the non-sepsis group, our method is applicable to other cases,

298 indicating that it may not depend on diagnoses in the ICU. Under intensive care

299 conditions, avoiding the occurrence of missing events is a challenge; therefore, our

300 method can be useful in the clinic. Distinguish a generalizable method from a

301 generalizable predictive model for clinical applications of machine learning is important.

302 This study shows availability of subgrouping based on missing events as a generalizable

303 method and production of a promising predictor model as a clinical decision support

304 tool. 
306 Conclusions

307 We developed a method to predict patient mortality based on information on

308 missing events. This method more accurately predicted patient mortality than others,

309 while maintaining clinical interpretability. Our results indicate that the subgrouping

310 process is important and effective for machine learning against patient heterogeneity.

311 By combining our method with other methods, such as the reinforcement learning, a

312 more realistic Artificial Intelligence clinician can be developed.

313

\section{List of abbreviations}

315 APACHE: Acute Physiology and Chronic Health Evaluation

316 ICU: Intensive Care Unit

317 eRI: eICU Research Institute

318 APS: Acute Physiology Score

319 ROC: Receiver Operating Characteristic

320 AUROC: Area Under the ROC 


\section{Ethics approval and consent to participate}

324 Not applicable.

325

326 Consent for publication

327 Not applicable.

329 Availability of data and materials

330 The datasets generated and/or analyzed during the current study are available in the

331 eICU repository, [https://eicu-crd.mit.edu/gettingstarted/access/].

\section{Competing interests}

334 The authors declare that they have no competing interests.

335

336 Funding

337 This work was supported by JSPS KAKENHI Grant Number JP 20K17834. 
340 ST analyzed the eRI data, suggested, and implemented subgrouping algorithm and

341 random forest model, prepared all of Figures and Tables and was a major contributor in

342 writing the manuscript. YH selected the 53 explanatory variables in the point of clinical

343 view, was the other major contributor in writing the manuscript as a clinician and

344 supported the research grant. SY checked the programs that ST wrote, reviewed the

345 manuscript and supported the research grant. KY contributed to the conception and

346 overall design of the work, reviewed the results and manuscripts as a clinical statistician

347 and supported the research grant. Finally, all four authors played an essential role in

348 organizing this project.

\section{Acknowledgments}

351 We thank Ryota Jin for discussions and comments to the manuscript.

\section{References}

354 1. Moons KG, Royston P, Vergouwe Y, Grobbee DE, Altman DG. Prognosis and

355 prognostic research: what, why, and how? BMJ. 2009;338:b375. 
356 2. Rudd KE, Johnson SC, Agesa KM, Shackelford KA, Tsoi D, Kievlan DR, et al.

357 Global, regional, and national sepsis incidence and mortality, 1990-2017:

358 analysis for the Global Burden of Disease Study. Lancet. 2020;395:200-11.

359 3. Seymour CW, Kennedy JN, Wang S, Chang CC, Elliott CF, Xu Z, et al.

360 Derivation, validation, and potential treatment implications of novel clinical

361 phenotypes for sepsis, J Am Med Assoc. 2019;321:2003.

362 4. Schinkel M, Paranjape K, Nannan Panday RS, Skyttberg N, Nanayakkara PWB.

363 Clinical applications of artificial intelligence in sepsis: a narrative review.

$364 \quad$ Comput Biol Med. 2019;115:103488.

365 5. Vincent JL, Moreno R. Clinical review: scoring systems in the critically ill. Crit

$366 \quad$ Care. 2010;14:207.

367 6. Knaus WA, Draper EA, Wagner DP, Zimmerman JE. APACHE II: a severity of

368 disease classification system. Crit Care Med. 1985;13:818-29.

369 7. Zimmerman JE1, Kramer AA. Outcome prediction in critical care: the Acute

370 Physiology and Chronic Health Evaluation models. Curr Opin Crit Care.

$371 \quad 2008 ; 14: 491-7$

372 8. Deo RC. Machine learning in medicine. Circulation. 2015;132:1920-30. 
373 9. Kourou K, Exarchos TP, Exarchos KP, Karamouzis MV, Fotiadis DI. Machine

374 learning applications in cancer prognosis and prediction. Comput Struct

$375 \quad$ Biotechnol J. 2015;13:8-17.

376 10. Breiman L. Random forests. Mach Learn. 2001;45:5-32.

377 11. Johnson AE, Pollard TJ, Shen L, Li-wei HL, Feng M, Ghassemi M, et al.

378 MIMIC-III, a freely accessible critical care database. Sci Data. 2016;3:160035.

379 12. Harris S, Shi S, Brealey D, MacCallum NS, Denaxas S, Perez-Suarez D, et al.

380 Critical Care Health Infor- matics Collaborative (CCHIC): data, tools and

381 methods for reproducible research: a multi-centre UK intensive care database.

$382 \quad$ Int J Med Inform. 2018;112:82-9.

383 13. Dybowski R, Weller P, Chang R, Gant V. Prediction of outcome in critically ill

384 patients using artificial neural network synthesised by genetic algorithm. Lancet.

$385 \quad 1996 ; 347: 1146-50$.

386 14. Jaimes F1, Farbiarz J, Alvarez D, Martínez C. Comparison between logistic

387 regression and neural networks to predict death in patients with suspected sepsis

388 in the emergency room. Crit Care. 2005;9:R150-6. 
389 15. Calvert J, Mao Q, Hoffman JL, Jay M, Desautels T, Mohamadlou H, et al. Using

390 electronic health record collected clinical variables to predict medical intensive

391 care unit mortality. Ann Med Surg (Lond). 2016;11:52-7.

392 16. Taylor RA, Pare JR, Venkatesh AK, Mowafi H, Melnick ER, Fleischman W, et

393 al. Prediction of in-hospital mortality in emergency department patients with

394 sepsis: a local big data-driven, machine learning approach. Acad Emerg Med.

$395 \quad 2016 ; 23: 269-78$.

396 17. Calvert J, Mao Q, Hoffman JL, Jay M, Desautels T, Mohamadlou H, et al. Using

397 electronic health record collected clinical variables to predict medical intensive

398 care unit mortality. Ann Med Surg (Lond). 2016;11:52-7.

399 18. Ward L, Paul M, Andreassen S. Automatic learning of mortality in a CPN model

400 of the systemic inflammatory response syndrome. Math Biosci. 2017;284:12-20.

401 19. Aushev A, Ripoll VR, Vellido A, Aletti F, Pinto BB, Herpain A, et al. Feature

402 selection for the accurate prediction of septic and cardiogenic shock ICU

403 mortality in the acute phase. PLoS One. 2018;13:e0199089.

404 20. Meiring C, Dixit A, Harris S, MacCallum NS, Brealey DA, Watkinson PJ, et al.

405 Optimal intensive care outcome prediction over time using machine learning.

$406 \quad$ PLoS One. 2018;13:e206862. 
407 21. García-Gallo JE, Fonseca-Ruiz NJ, Celi LA, Duitama-Muñoz JF. A machine

408 learning-based model for 1-year mortality prediction in patients admitted to an

409 Intensive Care Unit with a diagnosis of sepsis. Med Intensiva. 2020;44:160-70.

410 22. Pollard TJ, Johnson AE, Raffa JD, Celi LA, Mark RG, Badawi O. The eICU

411 Collaborative Research Database, a freely available multi-center database for

$412 \quad$ critical care research. Sci Data. 2018;5:1-13.

413 23. Pedregosa F, Varoquaux G, Gramfort A, Michel V, Thirion B, Grisel O, et al.

$414 \quad$ Scikit-learn: machine learning in python. J Mach Learn Res. 2011;12:2825-30.

415 24. Cortes C, Mohri M. Confidence intervals for the area under the ROC curve. Adv

$416 \quad$ Neural Inform Process Syst. 2015;17:305-12.

417 25. Mallinckrod CH, Lane PW, Schnell D, Peng Y, Mancuso JP. Recommendations

418 for the primary analysis of continuous endpoints in longitudinal clinical trials.

419 Drug Inf J. 2008;42:303-19.

420 26. Buuren SV, Groothuis-Oudshoorn K. mice: multivariate imputation by chained

421 equations in R. J Stat Softw. 2011;45:1-67. 

sepsis in the intensive care unit with minimal electronic health record data: a machine learning approach. JMIR Medical Informatics. 2016;4:e5909.

\section{Figure Legends}

\section{Fig. 1 Distribution of Acute Physiology Score (APS)-related variables.}

435 a The distribution of APS-related variables in the sepsis group. b Joint matrix for

436 checking the missing values. Correlation coefficients in some combinations were picked

437 up and visualized using the heatmap where higher coefficients were lighter and lower

438 were darker. 
440 Fig. 2 The performance of our model.

441 a Schematic of subgrouping results. The sepsis group was divided into five subgroups,

442 namely subgroup \#1 (884 cases), \#2 (1037 cases), \#3 (509 cases), \#4 (1230 cases), and

443 \#5 (566 cases). Colors indicate information about missing values. For example, cases in

444 subgroup \#1 have no missing values for all variables except "hospitaladmitoffset". b

445 Receiver operating characteristic (ROC) curves for each subgroup produced using our

446 model (red), Acute Physiology and Chronic Health Evaluation (APACHE) IV (green),

447 and APACHE IVa (blue). The integrated version is shown on extreme right. For the

448 confidence interval of AUROC, see Table S6 in Additional file 1. c Scatter plots of

449 predicted mortality; APACHE IV vs. APACHE IVa (left), APACHE IV vs. our model

450 (middle), and APACHE IVa vs. our model (right). Colors indicate actual mortality

451 (green for "ALIVE” and red for "EXPIRED” cases). For scatter plots of each subgroup,

452 see Fig. S2 in Additional file 2. d Calibration plots for our model (left), APACHE IV

453 (middle), and APACHE IVa (right). For the plot for each subgroup, see Fig. S3 in

454 Additional file 2. 


\section{Fig. 3 Performance of our model against the model based on the imputation}

\section{7 approach.}

458 a Receiver operating characteristic (ROC) curves for the four models, namely our

459 model (red), Acute Physiology and Chronic Health Evaluation (APACHE) IV (green),

460 APACHE IVa (blue), and imputation (orange). For the confidence interval of AUROC,

461 see Table S7 in Additional file 1. b Scatter plots of predicted mortality; imputation vs.

462 APACHE IV (left), imputation vs. APACHE IVa (middle), and imputation vs. our

463 model (right). Colors indicate actual mortality (green for "ALIVE" and red for

464 "EXPIRED" cases). c Calibration plots for the model based on the imputation approach.

\section{Fig. 4 Performance in the non-sepsis group.}

467 a Schematic image for the result of subgrouping. The non-sepsis group was divided into

468 seven subgroups, namely \#1 (3703 cases), \#2 (4112 cases), \#3 (1414 cases), \#4 (930

469 cases), \#5 (9487 cases), \#6 (786 cases), and \#7 (2738 cases). Colors indicate the

470 information about missing cases. For example, cases in the subgroup \#1 have no

471 missing values for all variables except "hospitaladmitoffset". b Receiver operating

472 characteristic (ROC) curve for each subgroup using our model (red), Acute Physiology

473 and Chronic Health Evaluation (APACHE) IV (green), and APACHE IVa (blue). The 
474 integrated version is shown on extreme right with the result from the imputation

475 approach. For the confidence interval of the area under the ROC (AUROC), see Table

$476 \quad$ S8 in Additional file 1.

477

478 Supplementary Information

479 Additional file 1

480 File name: Additional_file_1.xlsx

481 File format: Microsoft excel.

482 Title of data: Tables S1-S8.

483 Description of data: Supplementary tables.

484

485 Additional file 2

486 File name: Additional_file_2.pptx

487 File format: Microsoft power point.

488 Title of data: Figures S1-S5.

489 Description of data: Supplementary figures. 


\section{Fig. S1 Flowchart for generating subgroups.}

493 The blue box at the top indicates the starting point of the flowchart. Subgroups for the

494 sepsis or non-sepsis group are generated by following steps of the flowchart. The set $P$

495 is the defined patient list $(P=4226$ for the sepsis group and $P=23170$ for the

496 non-sepsis group). $V$ is the set of explanatory variables (i.e., the size of the $V$ is 53 ). $N_{\min }$

497 and $N_{\max }$ are set at 500 and 2000, respectively, for the sepsis group and 500 and 10000 ,

498 respectively, for the non-sepsis group. $2^{V}$ denotes the power set of set $V . V_{d j}^{c}$ denotes the

499 complementary subset for $V_{d j}$. $\mathrm{n}(A)$ denotes the size of set $A$.

500

501 Fig. S2 Scatter plot for the predicted mortality of each subgroup.

502 Scatter plots for predicted mortality; Acute Physiology and Chronic Health Evaluation

503 (APACHE) IV vs. APACHE IVa (left column), APACHE IV vs. our model (middle

504 column), and APACHE IVa vs. our model (right column). Colors indicate actual

505 mortality (green for “ALIVE” and red for "EXPIRED” cases).

506

507 Fig. S3 Calibration plots for each subgroup.

508 Calibration plots for our model (left column), Acute Physiology and Chronic Health

509 Evaluation (APACHE) IV (middle column), and APACHE IVa (right column). 
511 Fig. S4 Scatter plots of predicted mortality in the non-sepsis group.

512 Scatter plots of predicted mortality for each subgroup and integrated version; Acute

513 Physiology and Chronic Health Evaluation (APACHE) IV vs. APACHE IVa (left

514 column), APACHE IV vs. our model (middle column), and APACHE IVa vs. our

515 model (right column). Three panels at the bottom show the comparison with the model

516 generated based on the imputation approach. Colors indicate actual mortality (green for

517 “ALIVE" and red for "EXPIRED” cases).

519 Fig. S5 Calibration plots in the non-sepsis group.

520 Calibration plots in the non-sepsis group for each subgroup and the integrated version.

521 Columns on the left, middle, and right show results from our model, Acute Physiology

522 and Chronic Health Evaluation (APACHE) IV, and APACHE IVa, respectively. The

523 bottom panel shows results using the model generated based on the imputation

524 approach.

525

526 Supplementary Table Legends

527 Table S1 Working dataset used in this study. 
528 The column on the left corresponds to file names, which can be downloaded from the

529 eRI. We used 4 out of 31 files. The column in the middle shows the names of variables

530 selected in this study. The column on the right indicates the role of variables in this

531 study, where "key" indicates the unique key used for merging each .csv file and " $x$ " and

532 "y" indicate explanatory and response variables for machine learning, respectively,

533 Acute Physiology and Chronic Health Evaluation (APACHE) indicates predicting

534 patient mortality using APACHE IV or IVa, which was used as a benchmark against our

535 model, and "classification" indicates the variable that is used to define the sepsis or

536 non-sepsis group.

537

538 Table S2 Results from cross-validation for each subgroup from the sepsis group.

540 Table S3 Results from cross-validation for imputed data from the sepsis group.

542 Table S4 Results from cross-validation for each subgroup from the non-sepsis

543 group. 
545 Table S5 Results from cross-validation for imputed data from the non-sepsis

546 group.

547

548 Table S6 Confidence interval of area under the receiver operating characteristic

549 (AUROC) for each subgroup and the integrated version.

550

551 Table S7 Confidence interval of area under the receiver operating characteristic

552 (AUROC) for all four models.

553

554 Table S8 Confidence interval of area under the receiver operating characteristic

555 (AUROC) for each subgroup and the integrated version in the non-sepsis group. 
medRxiv preprint doi: https://doi.org/10.1101/2021.02.28.21252249; this version posted March 2, 2021. The copyright holder for this preprint (which was not certified by peer review) is the author/funder, who has granted medRxiv a license to display the preprint in perpetuity. All rights reserved. No reuse allowed without permission.

Figure 1

(A)
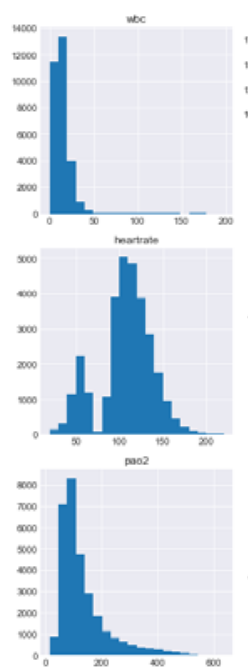
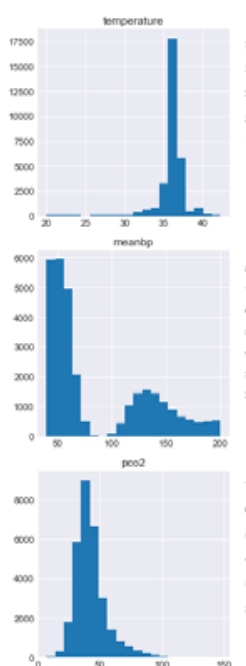
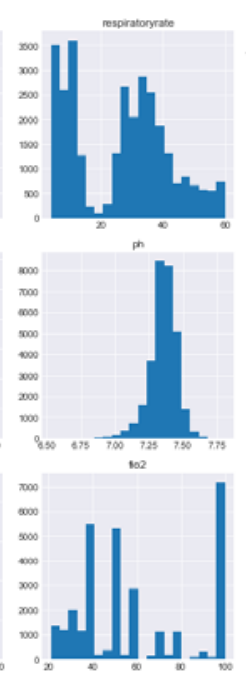

(B)
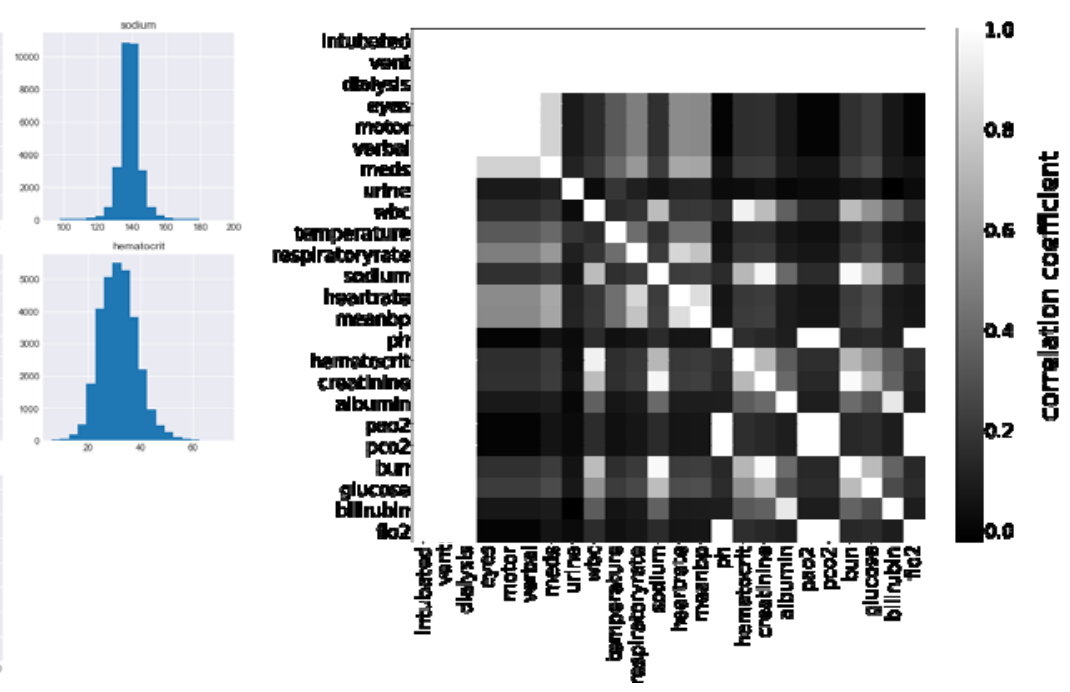
medRxiv preprint doi: https://doi.org/10.1101/2021.02.28.21252249; this version posted March 2, 2021. The copyright holder for this preprint All rights reserved. No reuse allowed without permission.

Figure 2

(A)

The sepsis group $(4,226$ cases)

53 variables

\begin{tabular}{|c|c|c|c|c|c|}
\hline Subgroups & $\# 1$ & $\# 2$ & \#3 & $\# 4$ & \#5 \\
\hline Variables & $\begin{array}{l}884 \\
\text { cases }\end{array}$ & $\begin{array}{l}1037 \\
\text { cases }\end{array}$ & $\begin{array}{c}509 \\
\text { cases }\end{array}$ & $\begin{array}{l}1230 \\
\text { cases }\end{array}$ & $\begin{array}{c}566 \\
\text { cases }\end{array}$ \\
\hline hospitaladmitoffset & & & & & \\
\hline $\begin{array}{ll}\text { urine } \\
\text { un }\end{array}$ & & & & & \\
\hline predventday & & & & & \\
\hline bilirubin and albumin & & & & & \\
\hline $\begin{array}{l}\text { age, creatinine, admissionweight } \\
\text { admissionheight, bun, glucose, }\end{array}$ & & & & & \\
\hline $\begin{array}{l}\text { The other parameters } \\
\text { (42 parameters) }\end{array}$ & & & & & \\
\hline
\end{tabular}

(B)

(C)
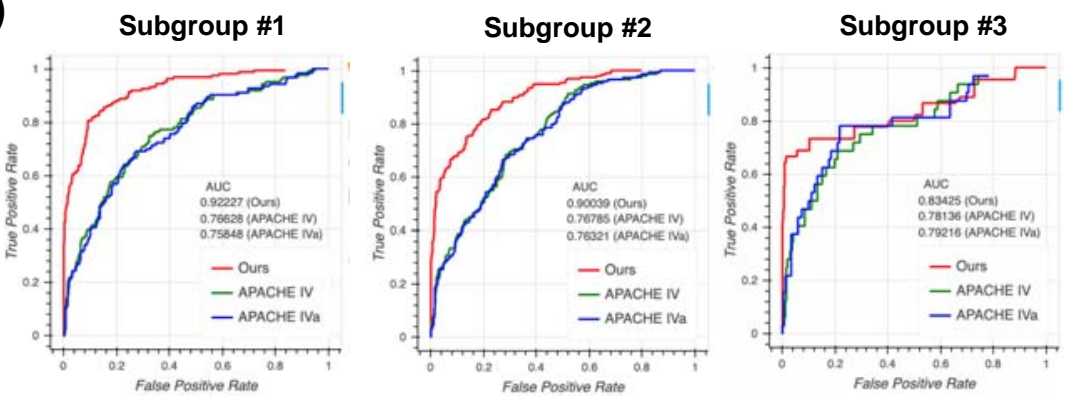

Subgroup \#4

Subgroup \#5
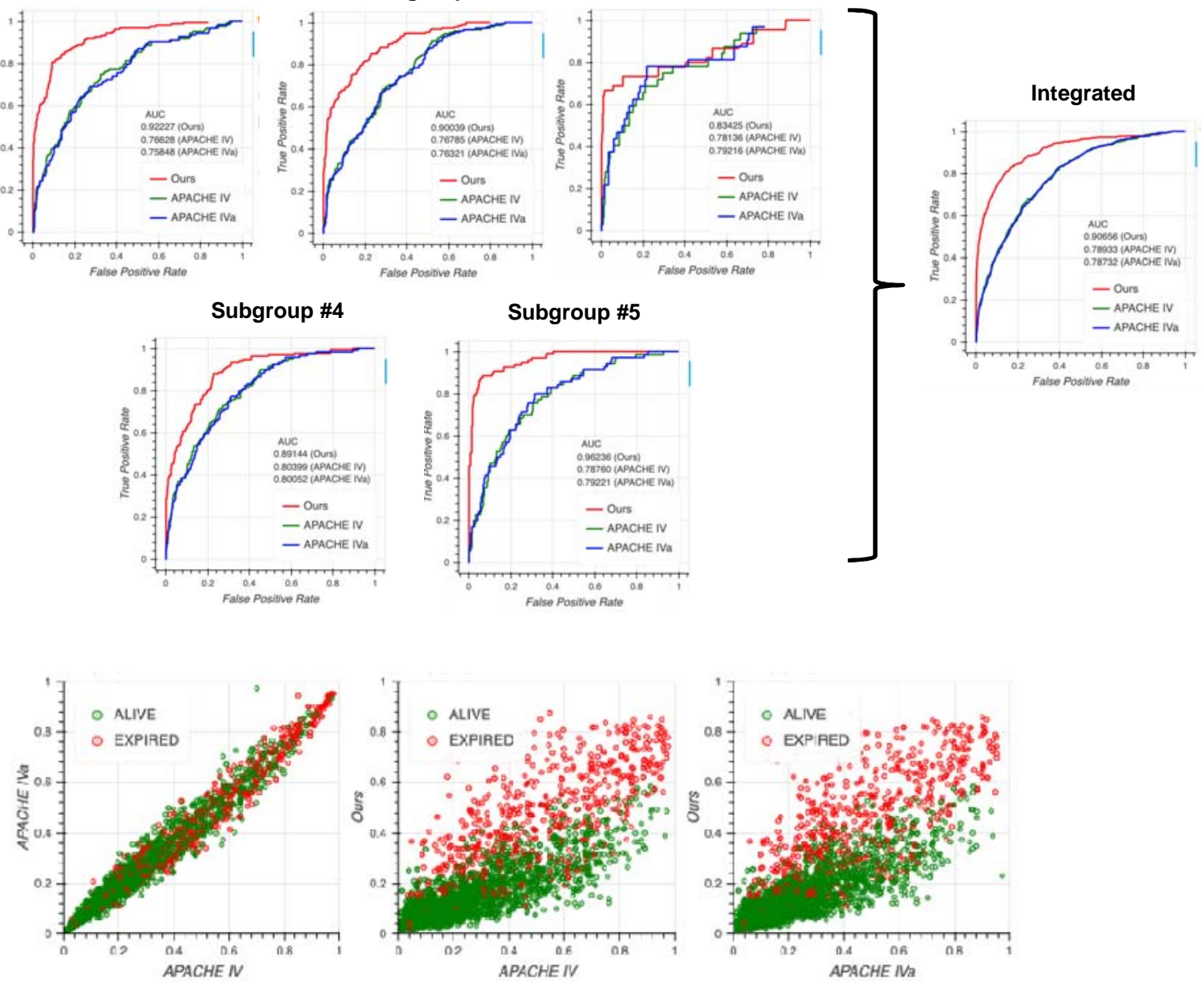

(D)

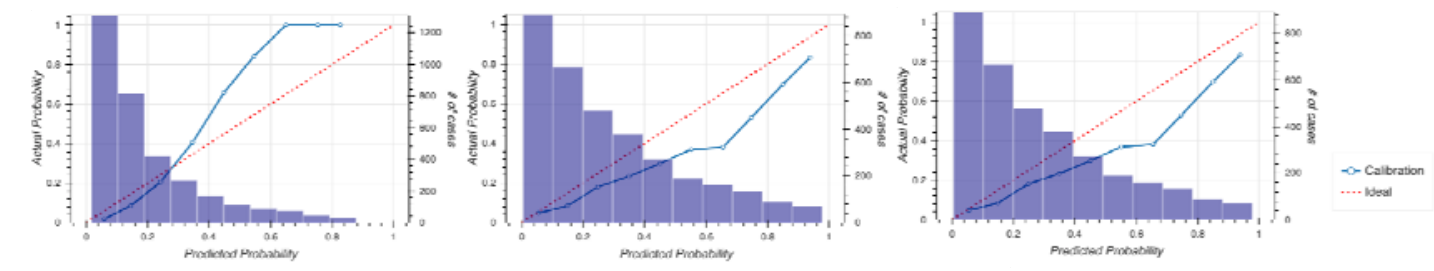


medRxiv preprint doi: https://doi.org/10.1101/2021.02.28.21252249; this version posted March 2, 2021. The copyright holder for this preprint (which was not certified by peer review) is the author/funder, who has granted medRxiv a license to display the preprint in perpetuity. All rights reserved. No reuse allowed without permission.

\section{Figure 3}

(A)

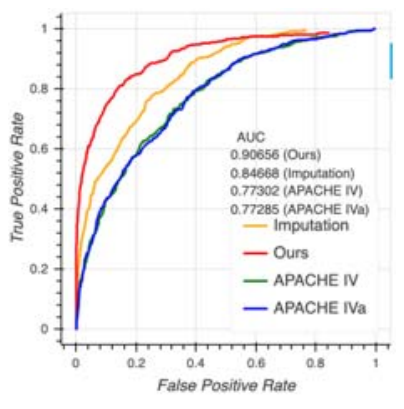

(B)

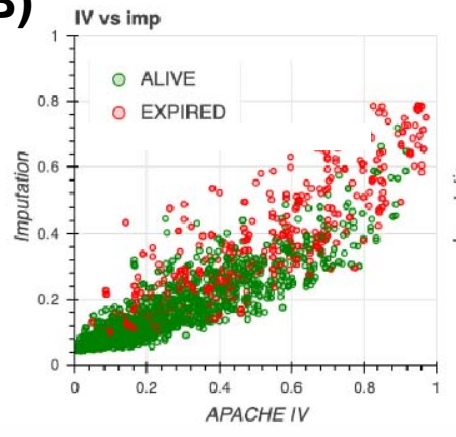

(C)
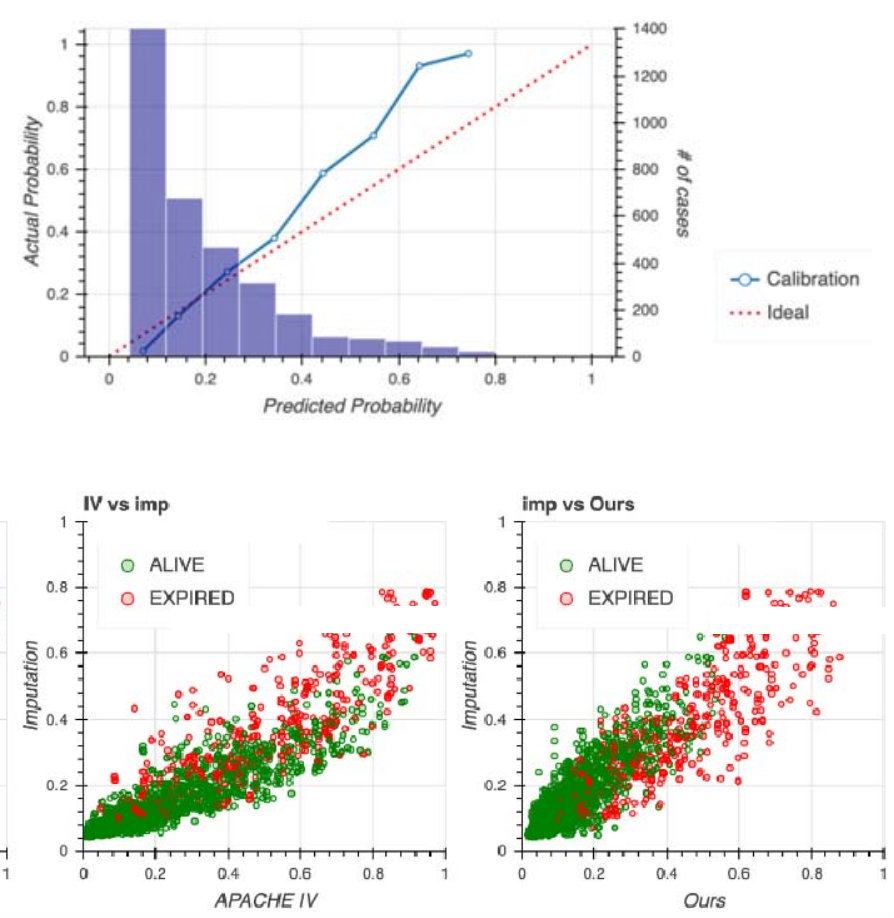
medRxiv preprint doi: https://doi.org/10.1101/2021.02.28.21252249; this version posted March 2, 2021. The copyright holder for this preprint (which was not certified by peer review) is the author/funder, who has granted medRxiv a license to display the preprint in perpetuity. All rights reserved. No reuse allowed without permission.

Figure 4

(A)

The non-sepsis group $(23,170$ cases)

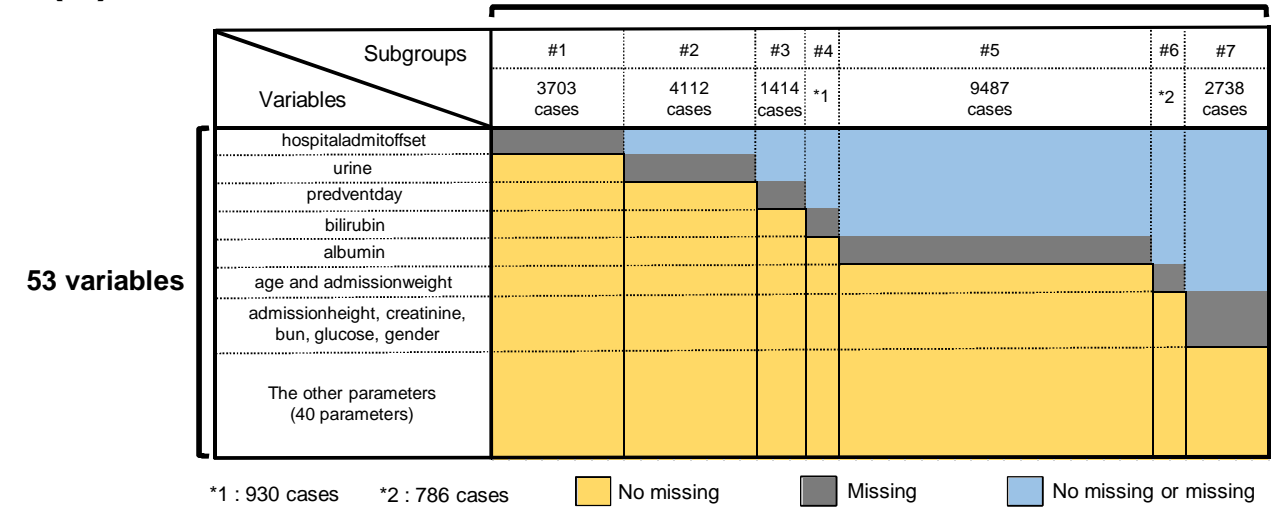

(B)
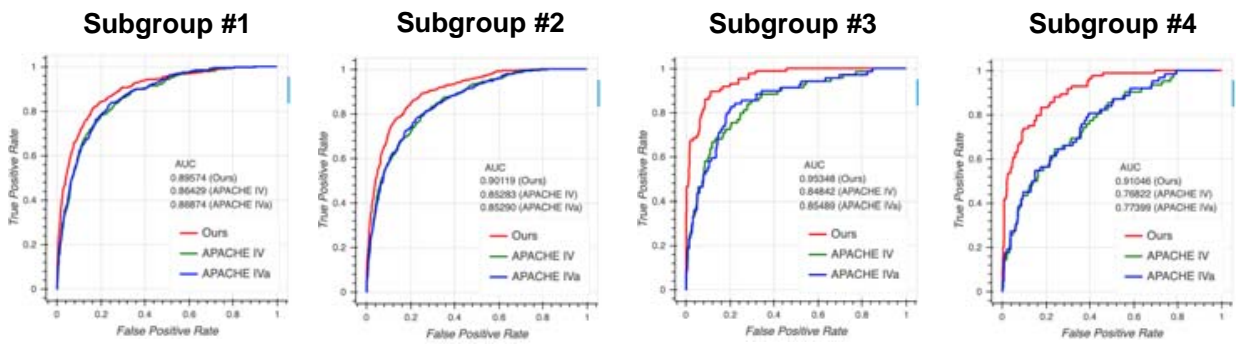

\section{Subgroup \#5}
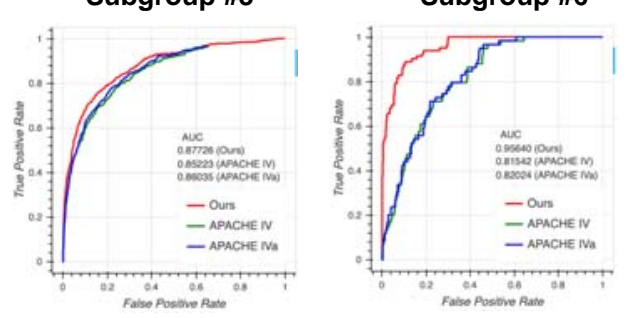

Subgroup \#7
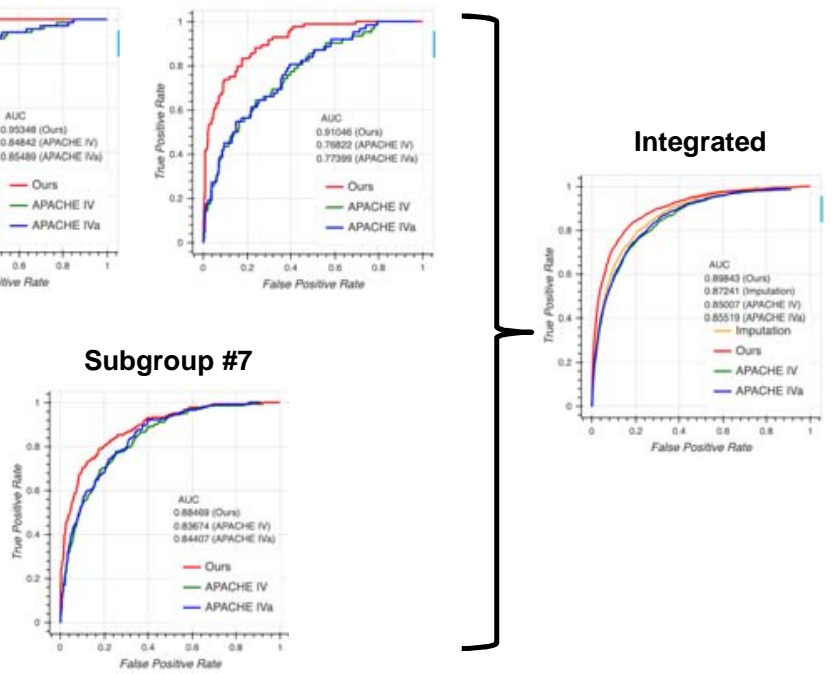